\title{
CORRELACIÓN ENTRE EL EXAMEN DE ADMISIÓN Y EL RENDIMIENTO EN EL PRIMER AÑO DE LA CARRERA ENSEÑANZA DE LA MATEMÁTICA EN LA UNA
}

\author{
Edwin Chaves ${ }^{1}$ \\ Director de la Escuela de Matemática de la Universidad Nacional \\ Heredia, Costa Rica \\ Mario Castillo ${ }^{2}$ \\ Suddirector de la Escuela de Matemática de la Universidad Nacional \\ Heredia, Costa Rica \\ Ronny Gamboa ${ }^{3}$ \\ Coordinador de la carrera Bachillerato y Licenciatura en la Enseñanza de la Matemática \\ de la Escuela de Matemática de la Universidad Nacional \\ Heredia, Costa Rica
}

Recibido: 5 de noviembre, 2007 - Aprobado: 15 de enero, 2008

Resumen: Este artículo plantea la correlación entre el examen de admisión de la Universidad Nacional de Costa Rica, respecto al rendimiento de los estudiantes de primer ingreso a la carrera Bachillerato y Licenciatura en la Enseñanza de la Matemática, en los primeros dos cursos de la especialidad.

En este análisis se consideran las variables: calificación en examen de admisión (total y por módulos), promedio de secundaria y rendimiento en los primeros dos cursos matemáticos de la carrera. Para determinar la relación entre las variables se utiliza el coeficiente de correlación de Pearson.

Los resultados revelan que los módulos del examen de admisión presentan una baja correlación, con respecto al rendimiento en dichos cursos. Por esta razón, pareciera que el actual proceso de selección de estudiantes de nuevo ingreso a esa carrera, no está realizando una adecuada discriminación con respecto a las bases necesarias para garantizar el éxito.

Palabras clave: Admisión a la universidad, examen de admisión, rendimiento académico, enseñanza de la Matemática, educación matemática.

Abstract: This article analyzes the relationship between the admission exams from the Universidad Nacional de Costa Rica (UNA) and the performance of students during their first two courses of specialization in the field of Teaching Mathematics.

In this analysis, the following variables were considered: individual result at the admission exam, average performance during high school and during the first two Mathematics courses of the career. To determine the relation between the variables, Pearson's correlation coefficient was used.

Doctor en Educación por la Universidad Estatal a Distancia. Máster en Estadística por la Universidad de Costa Rica. Licenciado en la Enseñanza de la Matemática por la Universidad Nacional. Profesor de la Escuela de Matemática de la UNA y de la Escuela de Estadística de la UCR. Actualmente es el director de la Escuela de Matemática de la UNA. echa@una.ac.cr

Doctor en Educación por la Universidad Estatal a Distancia. Máster en Estadística por la Universidad de Costa Rica. Egresado de la licenciatura en la Enseñanza de la Matemática por la Universidad Nacional. Profesor de la Escuela de Matemática de la UNA, Escuela de Salud Pública de la UCR y del Programa de Doctorado en Educación de la UNED. Actualmente es el suddirector de la Escuela de Matemática de la UNA mcastill@una.ac.cr

3. Máster en Matemática Educativa por el Centro de Investigación y Estudios Avanzados del Instituto Politécnico Nacional, México. Licenciado en la Enseñanza de la Matemática por la Universidad Nacional. Profesor de la Escuela de Matemática de la UNA. Actualmente es el coordinador de la carrera Bachillerato y Licenciatura en la Enseñanza de la Matemática. rgamboa@una.ac.cr 
The results reveal that the different modules of the admission exams show a low correlation with the performance in both courses. Therefore, the research concluded that the current process of selection of students, based on an admission exam, is not a tool that can be considered adequate to detect the previous knowledge to guarantee the success in the university career

Key words: Admission to the University, admissions exam, academic performance, teaching Mathematics, mathematical education.

\section{Introducción}

En la relación entre un estudiante con la universidad se pueden definir tres etapas que resultan de importancia dentro de las políticas universitarias: la primera consiste en los mecanismos de admisión o de ingreso; la segunda comprende la vida universitaria del estudiante (vida estudiantil) y la tercera corresponde a la finalización de la vida universitaria, que se puede dar por deserción o por graduación. Cada una de las etapas plantea problemas complejos que son objeto de debate y que constituyen cuestiones abiertas de estudio (Porto, Di Gresia y López, 2004). Este trabajo se ocupa de la primera etapa, la cual afecta, directa o indirectamente, las otras dos.

Mucho se ha pregonado sobre el derecho que tiene el ser humano a la educación. Se debe dar la oportunidad a cada persona de poder educarse en diferentes centros de educación formal: escuelas, colegios y universidades. No obstante, en la práctica, este derecho tiene sus límites, ya que es imposible pretender que todas las personas obtengan títulos universitarios, debido a que se produciría una saturación de profesionales en ciertos campos, y un déficit de recurso humano en una serie de actividades técnicas y de servicios que son fundamentales para la buena marcha de una sociedad (Tirado, Backhoof, Larrazolo y Rosas, 1997).

Tampoco se puede permitir que algunas personas permanezcan, perpetuamente, cursando estudios de una misma carrera, desperdiciando oportunidades de educación que podrían beneficiar a otros. El problema se acentúa en carreras que, como la enseñanza de la Matemática, tienen un grado de dificultad para un alto porcentaje de los estudiantes, lo que reduce las posibilidades de éxito de todos. Por esta razón, se requiere de un proceso de 
selección que contenga principios de justicia y equidad; $y$, al mismo tiempo, ofrezca una alta probabilidad de éxito a todos los estudiantes que logren ingresar a estas carreras. Una parte vital de este proceso consiste en evaluar las habilidades y los conocimientos básicos que poseen los alumnos que pretendan ingresar a la educación superior universitaria.

El proceso de admisión a la carrera Bachillerato y Licenciatura en la Enseñanza de la Matemática en la Universidad Nacional, ha pasado por diferentes etapas desde que se creó esta carrera, en 1974. En los últimos 10 años, se han utilizado diferentes modelos, entre ellos: la nota de presentación del colegio, la aplicación de un examen específico, entrevistas en profundidad, examen de admisión dentro de la Facultad de Ciencias Exactas y Naturales y, en los últimos años, el examen de admisión general, el cual se aplica a todo estudiante que desee ingresar a la Universidad Nacional. No obstante, pareciera que ninguno de estos instrumentos ha permitido favorecer el éxito a un alto porcentaje de los estudiantes que han sido admitidos. Este hecho queda evidenciado en los estudios realizados por Chaves (2003) y los datos sobre rendimiento académico de la Escuela de Matemática de esta universidad.

A pesar de que el mecanismo actual de selección de estudiantes, se ha aplicado en los últimos tres años, se han suscitado muchas dudas entre las autoridades y los docentes de la Escuela de Matemática, con respecto a la validez del mencionado proceso. Por esta razón, este estudio analiza la correlación entre los resultados de la prueba y el rendimiento de los estudiantes en los primeros cursos de la carrera Bachillerato y Licenciatura en la Enseñanza de la Matemática, específicamente en los cursos de matemática.

\section{Las pruebas de admisión}

En las instituciones universitarias de los países desarrollados se utilizan evaluaciones muy bien diseñadas, y que han superado fuertes procesos de validación. Por ejemplo, en los Estados Unidos se utiliza el Scholastic Aptitude Test (SAT) para ingresar a estudios de grado, el Graduate Record Examination (GRE) para posgrado y el Test of English as a Foreing Languaje (TOEFL) para acreditar el conocimiento de inglés como segundo idioma (Tirado et al., 1997). La Universidad 
de Costa Rica es la institución de educación superior, en este país, que cuenta con la mayor experiencia en este tipo de pruebas, pues realiza un examen de admisión, desde hace muchos años, a los estudiantes que desean ingresar a cursar un grado universitario, y ha presentado muy pocas variantes durante todo este tiempo.

Independientemente del procedimiento de admisión que aplique una institución para seleccionar a los estudiantes de nuevo ingreso, es fundamental valorar si dicho proceso toma en cuenta los elementos fundamentales relacionados con los objetivos institucionales. Además, un examen de admisión requiere de un análisis pscicométrico para determinar su validez. Magnusson (citado por Tirado et al., 1997) define la validez de un método como la exactitud con que pueden hacerse medidas significativas y adecuadas, en el sentido que midan los aspectos que se pretenden.

Por su parte, el College Board (1991) indica que la validez predictiva es una señal del grado hasta el cual las puntuaciones de un examen pueden predecir un criterio, el que se expresa como un coeficiente de correlación entre la variable predictiva y la variable criterio. En forma similar, Thorndike y Hagen (1996) definen la validez predictiva como la correlación entre las puntuaciones de una prueba y una medida de criterio adecuada.

Sin embargo, diversos especialistas en el tema evaluativo opinan que el poder lograr que una prueba tenga altos índices de validez no es una tarea fácil de conseguir. Además de los criterios mencionados anteriormente, para llevar a cabo una validación efectiva de una prueba, es necesario contar con medios alternativos que permitan triangular la información. Una manera de evaluar esta validez consiste en observar su potencialidad predictiva, es decir, determinar hasta dónde dicha prueba puede predecir el desenvolvimiento escolar futuro de los estudiantes. Al analizar la correlación entre las notas de secundaria, la nota en el examen de admisión a la Universidad Nacional respecto al rendimiento académico durante el primer año de la carrera, tanto en forma general como en sus diferentes módulos, se pretende determinar la capacidad predictiva del proceso de admisión a dicha carrera, que garantice el éxito en el desenvolvimiento del estudiante en los diferentes cursos del programa. 


\section{Metodología}

Como se ha venido señalando, se pretende determinar la correlación entre la prueba de admisión de la Universidad Nacional y el rendimiento académico en los primeros cursos de matemática de la carrera Bachillerato y Licenciatura en la Enseñanza de la Matemática. Puesto que se va a trabajar con la información previamente establecida, en la investigación no se lleva a cabo manipulación de las variables consideradas en el estudio. Por ello, el diseño de investigación es no experimental correlacional, de acuerdo con lo que plantean Hernández, Fernández y Baptista (2003).

Debido a que esta prueba de admisión se aplicó, por primera vez, para el ingreso al curso lectivo de 2005, el proceso investigativo involucró, únicamente, las generaciones de nuevo ingreso del 2005 y 2006.

Las principales variables consideradas en el estudio son:

a) Calificación promedio de secundaria (secund): corresponde a la nota de presentación de secundaria. Éste es un promedio obtenido en la educación diversificada.

b) Nota total en examen de admisión (adm_exa): resultado en la prueba de admisión en la Universidad Nacional.

c) Nota de admisión (adm_tot): representa la nota de admisión, la cual se obtiene de un 40\% de la nota del colegio (secund) y un $60 \%$ del examen de admisión (adm_exa), es decir: adm_tot $=0,4$ secund $+0,6$ adm_exa.

d) Nota obtenida en el curso de Matemática fundamental I (Funda_1): corresponde a la calificación obtenida en el curso MAB 300 Matemática fundamental I, durante el Primer Ciclo del curso lectivo.

e) Nota obtenida en el curso de Geometría euclídea I (Geome_1): corresponde a la calificación obtenida en el curso MAB 301 Geometría euclídea I, durante el Primer Ciclo del curso lectivo. 
f) Nota obtenida en el curso de Matemática fundamental II (Funda_2): corresponde a la calificación obtenida en el curso MAB 302 Matemática fundamental II, durante el Segundo Ciclo del curso lectivo.

g) Nota obtenida en el curso de Geometría euclídea II (Geome_2): corresponde a la calificación obtenida en el curso MAB 303 Geometría euclídea II, durante el Segundo Ciclo del curso lectivo.

Como el examen de admisión está constituido por diferentes módulos, también se consideran la calificación en estos módulos como variables secundarias.

a) Nota obtenida en el módulo de razonamiento abstracto (raz_abst).

b) Nota obtenida en el módulo verbal (verbal).

c) Nota obtenida en el módulo matemático (matema).

d) Nota obtenida en el módulo científico naturalista (cien_nat).

e) Nota obtenida en el módulo espacial perceptivo (esp_perc).

Las variables Funda_1, Funda_2, Geome_1 y Geome_2 fungirán como variables dependientes, mientras que las restantes serán consideradas como variables independientes.

La relación entre las variables se midió, fundamentalmente, por las correlaciones entre las variables. Para ello se utilizó el coeficiente de correlación de Pearson (Pardo y Ruiz, 2002). No obstante, también se emplearon modelos de regresión lineal, con el objetivo de valorar la capacidad predictiva de las variables independientes sobre las dependientes (Pardo y Ruiz). Para efectuar este análisis, se planteó el supuesto de que la información utilizada correspondía a datos aleatorios y se utilizó el software estadístico SPSS (Statistical Package for the Social Sciences). 


\section{Resultados}

De acuerdo con los registros de la Escuela de Matemática, para el año 2005, 87 estudiantes de primer ingreso a la universidad matricularon los primeros cursos de la carrera Bachillerato y Licenciatura en la Enseñanza de la Matemática y, para el año 2006, esta cifra aumentó a 89. Por ello, el estudio se concentró en un total de 176 estudiantes. En el estudio no se consideraron repitientes, ni estudiantes que ingresaron por cambio de carrera dentro de la misma universidad.

Un aspecto de gran relevancia consistió en que algunos de estos estudiantes se retiraron de los cursos después de pocos días de iniciar el curso lectivo; pero, no se conocen las razones que les motivó a retirarse. La Tabla № 1 resume la información básica de todos los estudiantes, de acuerdo con el año en que ingresaron a la carrera.

Tabla № 1

Distribución de los estudiantes de nuevo ingreso a la carrera Bach. y Lic. en la Enseñanza de la Matemática, según rendimiento académico en los cursos introductorios. 2005-2006

Año de ingreso

\begin{tabular}{|c|c|c|c|c|c|c|}
\hline & \multicolumn{2}{|c|}{2005} & \multicolumn{2}{|c|}{2006} & \multicolumn{2}{|c|}{ Total } \\
\hline & Funda_1 & Geome_1 & Funda_1 & Geome_1 & Funda_1 & Geome_1 \\
\hline Matriculados & 74 & 74 & 75 & 71 & 149 & 145 \\
\hline Retirados & 13 & 13 & 14 & 18 & 27 & 31 \\
\hline Nota promedio & 5,14 & 5,14 & 5,25 & 3,42 & 5,19 & 4,30 \\
\hline Nota mediana & 5,00 & 5,00 & 5,50 & 2,50 & 5,00 & 4,00 \\
\hline Desviación estándar & 2,09 & 2,20 & 2,01 & 2,29 & 2,05 & 2,40 \\
\hline$\%$ de aprobación ${ }^{1}$ & 29,7 & 35,1 & 32,0 & 16,9 & 30,9 & 26,2 \\
\hline
\end{tabular}

* $\quad$ No considera los retirados 
Los estudiantes retirados se descartaron de los principales análisis subsiguientes, aunque se debe destacar que el porcentaje de retiros es muy alto (cercano al 20\%). Alrededor de un $30 \%$ de los estudiantes logró aprobar el curso Matemática fundamental (MAB 300), mientras que, en Geometría (MAB 301), el porcentaje de aprobación tuvo un fuerte descenso en el 2006, con respecto al 2005. Independientemente de estas diferencias, estos resultados reflejan problemas con respecto al desarrollo de los cursos introductorios a esta carrera, pues el rendimiento es muy bajo, el porcentaje medio de aprobación ronda el 30\%.

Muchos factores pueden estar asociados con estos resultados; uno de ellos podría estar relacionado con el proceso de selección de estudiantes de nuevo ingreso. Por esta razón, es importante analizar la correlación entre los resultados obtenidos con los que se utilizan para la selección de estudiantes.

En primer lugar, se analizan las calificaciones obtenidas en el proceso de admisión de estos estudiantes, así como su nota promedio de Educación diversificada. La Tabla № 2 muestra estos resultados.

\section{Tabla № 2}

Distribución de los estudiantes de nuevo ingreso a la carrera Bach. y Lic. en la Enseñanza de la Matemática, según la nota de admisión y la calificación promedio en la Educación diversificada. 2005-2006

\begin{tabular}{|c|c|c|c|c|c|c|}
\hline & \multicolumn{6}{|c|}{ Año de ingreso } \\
\hline & \multicolumn{3}{|c|}{2005} & \multicolumn{3}{|c|}{2006} \\
\hline & secund & adm_exa & adm_tot & second & adm_exa & adm_tot \\
\hline Total de estudiantes & 87 & 87 & 87 & 89 & 89 & 89 \\
\hline Nota promedio & 86,2 & 59,0 & 69,9 & 86,4 & 60,4 & 70,7 \\
\hline Nota mediana & 86,0 & 59,2 & 69,5 & 86,2 & 60,0 & 69,7 \\
\hline Desviación estándar & 5,7 & 6,3 & 4,2 & 6,2 & 6,4 & 4,49 \\
\hline
\end{tabular}

Esta información no muestra importantes diferencias entre los resultados de un año a otro. Desde un punto de vista estadístico, bajo el supuesto de que los datos corresponden a muestras aleatorias, las diferencias obtenidas entre los años 2005 y 2006 no son significativas al nivel del 5\%, para las variables secund, adm_exa y adm_tot. 
Por otro lado, debido a que la nota máxima en el examen de admisión es 100, puede notarse que los estudiantes que ingresaron a la carrera Enseñanza de la Matemática tienen una calificación relativamente baja, y una variabilidad relativamente alta. Éste es un elemento por tomar en cuenta al analizar el rendimiento en los cursos de matemática.

El coeficiente de correlación entre la nota promedio de la educación diversificada (secund) y la nota del examen de admisión (adm_exa) es apenas de -0,081, por lo que la calificación del examen de admisión no parece estar relacionada con la nota promedio de la educación diversificada.

La correlación entre estas variables secund, adm_exa y adm_tot, con respecto a las calificaciones de los cursos introductorios Funda_1 y Geome_1, se muestra en la Tabla № 3.

Tabla № 3

\begin{tabular}{|c|c|c|c|c|c|}
\hline & \multicolumn{5}{|c|}{$\begin{array}{l}\text { Matriz de correlación de Pearson de las variables } \\
\text { secund, adm_exa, adm_tot, Funda_1 y Geome_1 }\end{array}$} \\
\hline & secund & adm_exa & adm_tot & Funda_1 & Geome_1 \\
\hline secund & 1,00 & 0,08 & $0,48^{*}$ & $0,33^{*}$ & $0,31^{*}$ \\
\hline adm_exa & & 1,00 & $0,84^{*}$ & $0,26 *$ & 0,16 \\
\hline adm_tot & & & 1,00 & $0,40 *$ & $0,30 *$ \\
\hline Funda_1 & & & & 1,00 & $0,68^{*}$ \\
\hline Geome_1 & & & & & 1,00 \\
\hline
\end{tabular}

* Las correlaciones son significativas al nivel del $1 \%$

Los valores son sumamente bajos. En las últimas dos columnas se pueden determinar las relaciones de interés para el estudio. Es preocupante que las correlaciones más bajas se establecen entre la nota del examen de admisión y las calificaciones obtenidas en los cursos introductorios a la carrera. No obstante, las correlaciones con el promedio de secundaria son, también, bajas y, aunque son significativas, estadísticamente, tienen muy poco valor predictivo. La Tabla № 4 presenta las correlaciones para cada uno de los años en estudio. 
Tabla № 4

Correlaciones de Pearson de las variables secund, adm_exa, adm_tot, Funda_1 y Geome_1, años 2005 y 2006

\begin{tabular}{lcccc}
\hline & \multicolumn{2}{c}{2005} & \multicolumn{2}{c}{2006} \\
\hline & Funda_1 & Geome_1 & Funda_1 & Geome_1 \\
\hline secund & 0,30 & $0,41^{*}$ & $0,36^{*}$ & $0,27^{*}$ \\
adm_exa & 0,17 & 0,09 & $0,35^{*}$ & $0,34^{*}$ \\
adm_tot & $0,31^{*}$ & 0,29 & $0,49^{*}$ & $0,44^{*}$ \\
Funda_1 & 1,00 & $0,79^{*}$ & 1,00 & $0,70^{*}$ \\
Geome_1 & $0,79^{*}$ & 1,00 & $0,70^{*}$ & 1,00 \\
\hline
\end{tabular}

* Las correlaciones son significativas al nivel del $1 \%$.

Aunque existen diferencias entre los años 2005 y 2006, los patrones de la Tabla № 3 se repiten en la anterior. El hecho que más llama la atención, consiste en la baja correlación que muestra el examen de admisión con respecto a Funda_1 y Geome_1, durante el año 2005, lo que provoca que disminuya también la correlación de la nota de admisión total, con respecto a estos cursos. Esta situación revela que, para el 2005, el examen de admisión no discriminó con respecto a la selección de estudiantes para el ingreso a esta carrera. También se evidencia la inconsistencia de los resultados del examen de admisión entre un año y otro; sin embargo, la calificación promedio de la Educación diversificada, mantiene una mayor consistencia en esta correlación con los resultados obtenidos en los cursos de primer ingreso. Por otro lado, a lo interno de los dos cursos analizados, el coeficiente de correlación es aceptable, más de 0,70.

El examen de admisión está constituido por cinco módulos. Resulta de interés para este estudio, determinar cuáles de estos módulos presentan la mayor correlación con respecto a los cursos Funda_1 y Geome_1. La Tabla № 5, presenta la matriz de correlación entre los diferentes módulos. 
Tabla № 5

Matriz de correlación de Pearson entre los módulos del examen de admisión y los cursos Funda_1 y Geome_1. Período 2005-2006

\begin{tabular}{lccccccc}
\hline & verbal & raz_abst & matema & cien_nat & esp_perc & Funda_1 & Geome_1 \\
\hline verbal & 1,00 & 0,12 & 0,09 & 0,06 & 0,09 & $0,29 *$ & 0,16 \\
raz_abst & & 1,00 & 0,12 & 0,05 & $0,23^{*}$ & 0,08 & 0,16 \\
matema & & & 1,00 & 0,06 & 0,045 & $0,27^{*}$ & $0,34^{*}$ \\
cien_nat & & & & 1,00 & 0,070 & 0,14 & 0,03 \\
esp_perc & & & & & 1,00 & 0,06 & 0,02 \\
Funda_1 & & & & & & 1,00 & $0,68^{*}$ \\
Geome_1 & & & & & & 1,00 \\
\hline
\end{tabular}

* Las correlaciones son significativas al nivel del $1 \%$.

Puede notarse que, únicamente, los módulos verbal y matemático presentan una correlación significativa, con respecto a los cursos de primer nivel, aunque dicho coeficiente es muy bajo, por lo que podría decirse que la correlación presente es bastante débil. La Tabla № 6 complementa la información presentada en la anterior, dado que muestra los coeficientes de correlación en cada módulo del examen de admisión, con los cursos Funda_1 y Geome_1 para los años 2005 y 2006.

Tabla № 6

Correlación de Pearson entre los módulos del examen de admisión y los cursos Funda_1 y Geome_1, por año. Años 2005 y 2006

\begin{tabular}{lcccc}
\hline \multicolumn{1}{c}{ Módulo } & \multicolumn{2}{c}{2005} & & 2006 \\
\hline & Funda_1 & Geome_1 & Funda_1 & Geome_1 \\
\hline verbal & 0,22 & 0,11 & $0,36^{*}$ & $0,30^{*}$ \\
raz_abst & 0,14 & 0,27 & 0,01 & 0,06 \\
matema & 0,23 & 0,22 & $0,33^{*}$ & $0,40^{*}$ \\
cien_nat & 0,01 & 0,01 & 0,23 & 0,14 \\
esp_perc & 0,10 & 0,15 & 0,00 & 0,02 \\
\hline
\end{tabular}

* $\quad$ Las correlaciones son significativas al nivel del $1 \%$. 
Los módulos verbal y matemático son los que, mayormente, se correlacionan con los cursos Funda_1 y Geome_1. Aunque la correlación es muy débil, especialmente, para el 2005. Quizá, el aspecto más relevante se presenta con los resultados del módulo de razonamiento abstracto, pues debido a la naturaleza de la carrera, se esperaba que este módulo ofreciera un importante aporte al proceso de selección de estudiantes. No obstante, las correlaciones que se presentan en las Tablas № 5 y № 6 reflejan que, prácticamente, no existe dicha relación; pero, además, su signo negativo denota una contradicción con lo esperado. Por ello, se podría indicar que dicho módulo, no solamente no aporta elementos positivos para discriminar estudiantes que podrían tener un buen rendimiento en la carrera, sino que, también, pareciera que podría producir un efecto distorsionador.

\section{Hallazgos}

El proceso de admisión es un procedimiento muy complejo y delicado, debido a que, mediante este recurso, la Universidad decide cuáles estudiantes podrán optar por la educación estatal, ellos se convierten en la materia prima del quehacer de la Institución. Además de la selección estudiantil, este proceso establece las bases sobre el conocimiento del aspirante, con respecto a sus potencialidades, limitaciones, áreas por mejorar y, particularmente, las posibilidades de éxito en su trayectoria universitaria. Por esta razón, la información recabada en el proceso de admisión, permitirá a la Universidad orientar al estudiante de nuevo ingreso en su trayectoria dentro de la Institución, y establecer estrategias para culminar su formación profesional satisfactoriamente.

Ante los problemas que debe enfrentar la educación matemática en el país, es fundamental contar con un proceso eficiente de selección de estudiantes de nuevo ingreso a las carreras de Enseñanza de la Matemática, de modo que pueda establecer un potencial básico que garantice el éxito académico a los estudiantes que ingresen a estos programas y, de cara al futuro, se obtengan profesionales con una buena preparación en este campo, capaces de enfrentar los retos existentes. Por esta razón, se ha considerado oportuno determinar si el 
examen de admisión de la Universidad Nacional permite realizar este proceso en forma armoniosa.

El estudio arrojó resultados muy negativos. En primer lugar, se tiene una alta tasa de reprobación en los cursos de primer ingreso a la carrera. Del total de estudiantes admitidos durante el 2005 y 2006, más del 15\% se retiraron en las primeras semanas del curso lectivo, y, de los restantes, únicamente cerca de un $30 \%$ aprobó los primeros dos cursos de la especialidad. Por ello, un alto porcentaje de jóvenes ve frustradas sus aspiraciones iniciales de convertirse en profesor de Matemática y, aunque muchos repiten los cursos el año siguiente, se denota un serio problema en cuanto al éxito del proceso.

Por otro lado, al analizar la relación existente entre los resultados del examen de admisión con respecto a las calificaciones de los estudiantes en los cursos de matemática de primer ingreso, se ha encontrado una serie de contradicciones. En primer lugar, se determinó inconsistencia entre los resultados del examen en los años 2005 y 2006, con respecto al rendimiento académico de los estudiantes en los cursos Matemática fundamental 1 y Geometría euclídea 1. La correlación es muy débil; si se analiza el grado de explicación que tiene el examen de admisión con respecto a la calificación en los primeros cursos de la carrera, para el año 2005 es, prácticamente, nula y para el 2006 es, apenas, cercana a un 6\%. En general, la nota promedio de la Educación diversificada resulta un mejor parámetro o, al menos, más consistente entre un año y otro.

En cuanto al comportamiento de los cinco módulos del examen de admisión, dentro de las autoridades de la Escuela de Matemática, se tenía el supuesto que los módulos que se correlacionarían, en mayor medida, con el perfil necesario para la selección de los estudiantes para esta carrera, serían el matemático y el de razonamiento abstracto. No obstante, los resultados revelan que este último módulo presentó una correlación, prácticamente, nula con el rendimiento en los cursos de primer nivel y, en contradicción con lo esperado, su signo fue negativo. El módulo verbal presentó una correlación similar a la del módulo matemático, con respecto a los cursos del primer nivel de la carrera; pero ambos presentan un bajo coeficiente de correlación con dichos cursos. 
En síntesis, en los resultados obtenidos para los años 2005 y 2006, el examen de admisión no pareciera estar aportando elementos positivos en el proceso de selección de estudiantes de primer ingreso a la carrera Bachillerato y Licenciatura en la Enseñanza de la Matemática. Tampoco pareciera contribuir con elementos diferentes a los que aporta la nota promedio de la educación diversificada.

Por esta razón, si se desea incrementar la promoción en los cursos de primer ingreso, así como disminuir la deserción estudiantil durante el primer año, se requiere revisar, seriamente, el proceso de selección de estudiantes que serán admitidos cada año. De esta manera, se esperaría que dicho proceso fuera congruente para establecer los requerimientos mínimos que debe cumplir un estudiante para garantizar el éxito en sus estudios.

Pero, además, en las actuales circunstancias, es necesario que los responsables del plan de estudios de esta carrera, consideren la demanda estudiantil que, en la actualidad presenta este programa, sobre todo, en función de las bases académicas con que cuentan los estudiantes provenientes de secundaria. En los últimos años, se ha señalado, de manera, insistente, que estas bases se han deteriorado. Esta situación obliga a los responsables de las carreras universitarias a establecer un puente entre la secundaria y la universidad, de manera que el sistema universitario no rechace "ad portas" a los jóvenes, sin siquiera darles la oportunidad de incursionar en el proceso. En este sentido, existe una creciente tendencia a que las instituciones deben adaptar, cada vez más, los procesos al estudiante, en lugar de pretender que sea el estudiante el que se ajuste a los procesos preexistentes.

Por lo anterior, debería considerarse la admisión como un proceso que tiene una doble finalidad: por un lado, seleccionar a los alumnos de nuevo ingreso a la carrera con los mejores elementos de juicio y, por otro, como un mecanismo para conocer el perfil de ingreso de los jóvenes en forma individual y general. De esta manera, se logrará determinar, con mayor precisión, cuáles son las características fundamentales de los nuevos estudiantes. Esto permitirá estimular aquellas que puedan tener un efecto positivo en su desempeño académico, y, por ende, que le permitan enfrentar el estudio con mayores posibilidades de éxito. Pero, además, permite establecer estrategias para favorecer los cambios necesarios en aquellas características de los jóvenes, que tengan un efecto negativo para el proceso en el cual están incursionando. 
En general, los procesos de admisión deberían enfocarse en conocer las características del futuro estudiante universitario. Esto permitirá, no sólo seleccionarlo, sino también adquirir información precisa que permita, a cada unidad académica, orientar al joven durante el proceso educativo y diseñar, junto a otros aspectos, el currículo, en condiciones que sean las más favorables para ambos. Además, velar porque los procesos de generación de conocimiento, se lleven a cabo mediante estrategias novedosas que permitan, a los estudiantes, incorporarse, paulatinamente, al proceso e ir adquiriendo la madurez necesaria que, una vez concluidos sus estudios, le garanticen el éxito en su vida profesional. Por esta razón, la valoración de la admisión no debería depender sólo de un examen de conocimientos y habilidades, que se mide en un único momento, como ocurre, actualmente, en la UNA, sino que se requiere de un verdadero proceso que involucre varias etapas, mediante las cuales se obtenga más información sobre el aspirante.

\section{Referencias}

College Board. (1991). Guía de orientaciones y oficiales de admisión: prueba de aptitud académica y pruebas de aprovechamiento académico. San Juan, Puerto Rico: Autor.

Chaves, E. (2003). Graduación y deserción en la Escuela de Matemática de la UNA: Cohortes 1995 a 1998. Revista Uniciencia, 20 (1), 115-122.

Hernández, R.; Férnández, C. y Baptista, P. (2003). Metodología de la investigación. (3 ${ }^{\underline{a}}$ ed.). México: Editorial McGraw-Hill.

Pardo, A. y Ruiz, M. (2002). SPSS 11: Guía para el análisis de datos. Madrid: McGraw-Hill Interamericana.

Porto, A.; Di Gresia, L. y López, M. (2004). Mecanismos de admisión a la universidad y rendimiento de los estudiantes. Facultad de Ciencias Económicas, Universidad de La Plata, Argentina. Recuperado en 20 de abril de 2007, de www.depeco.econo.unlp.edu.ar/semi/semi100904.pdf

Thorndike, R. y Hagen, E. (1996). Medición y evaluación en psicología y educación. México: Trillas. 
Tirado, F.; Backhoff, E.; Larrazolo, N. y Rosas, M. (1997). Validez predictiva del examen de habilidades y conocimientos básicos (EXHCOBA). Revista Mexicana de Investigación educativa, 2(1), 67-84. 